\title{
Leukocytoclastic Vasculitis: A Retrospective Analysis of 57 Cases
}

\author{
Nermin Karaosmanoğlu' (1), Pınar Özdemir Çetinkaya ${ }^{2}$ (D), Engin Karaaslan ${ }^{3}$ (1) \\ 'Clinic of Dermatology and Venereology, Ankara Training and Research Hospital, Ankara, Turkey \\ ${ }^{2}$ Clinic of Dermatology and Venereology, Nazilli State Hospital, Aydın, Turkey \\ ${ }^{3}$ Clinic of Dermatology and Venereology, Nusaybin State Hospital, Mardin, Turkey
}

ORCID IDs of the authors: N.K. 0000-0002-3462-1628; P.ö.C̣. 0000-000I-5286-3260; E.K. 0000-0003-29II-5082.

Cite this article as: Karaosmanoğlu N, Özdemir C̣etinkaya P, Karaaslan E. Leukocytoclastic Vasculitis: A Retrospective Analysis of 57 Cases. Cyprus J Med Sci 2019; 4(3): 242-6.

\section{BACKGROUND/AIMS}

Cutaneous small vessel vasculitis (CSVV) is a small vessel vasculitis affecting the skin primarily, but may be associated with larger systemic vasculitis or extra cutaneous involvement. The clinical hallmark is palpable purpura usually involving the lower extremities. Although CSV $V$ can be induced by drugs, infections, connective tissue diseases, and malignancies, it can also be idiopathic. The aim of the present study was to analyze patients with cutaneous leukocytoclastic vasculitis (LCV) to determine disease characteristics.

\section{MATERIALS and METHODS}

In this retrospective study, the records of 57 patients with LCV from 2013 to 2017 managed at the Department of Dermatology were reviewed. A detailed evaluation of the data was done to determine demographic characteristics, etiological factors, clinical signs and symptoms, and treatment options.

\section{RESULTS}

The study included 39 female and 18 male patients. The median age of the patients was 55 years. The majority of patients (46, 80.7\%) were classified as CSVV. Almost half of the cases $(25,43.9 \%$ ) were idiopathic, but the most common cause was simultaneous infection and drug usage $(12,21 \%)$. The most common cutaneous manifestation was palpable purpura $(21,36.8 \%)$ involving the lower extremities $(40,70.2 \%)$. The most common extra cutaneous manifestation was joint involvement presenting as arthritis and/or arthralgia (15, 26.3\%). The most frequent laboratory abnormality was an elevated C-reactive protein (40, $70.2 \%)$.

\section{CONCLUSION}

In clinical practice, it is important to keep in mind that although LCV is usually confined to the blood vessels of the skin, a detailed evaluation of the patients is required to detect the underlying causes and systemic involvement.

Keywords: Leukocytoclastic vasculitis, small vessel vasculitis, vasculitis

\section{INTRODUCTION}

Vasculitides are a spectrum of diseases with a wide variety of clinical manifestations characterized by a specific inflammation of the blood vessels. The specific inflammation leads to destruction and subsequent hemorrhagic features (I). These conditions may be confined only to the skin or to a lesser extent may be a cutaneous sign of a systemic vasculitic syndrome which is also associated with multiple extracutaneous end organ involvements.

Over the years, various criteria and nomenclature systems have been developed to classify vasculitic syndromes. Unfortunately, there is no ideal system for the accurate classification of vasculitides. The two main classification systems in use are the American College of Rheumatology classification criteria and the revised Chapel Hill Consensus Conference nomenclature system $(2,3)$. In dermatology practice, it is more useful to classify cutaneous vasculitides based on involved vessel-size predominance (I). Vasculitis can be classified according to the following diameter of the involved vessel: (I) large vessels, (2) medium-sized vessels, and (3) small vessels including arterioles, capillaries, and specifically postcapillary venules (I).

The new name for a group of small vessel vasculitides, formerly called leukocytoclastic vasculitis (LCV), has been proposed to be cutaneous small vessel vasculitis (CSVV). LCV has been accepted as the name of a histological term which 
defines perivascular inflammatory infiltrate consisting of mainly neutrophils, fibrinoid necrosis, swelling of endothelial cells, and erythrocyte extravasation in the postcapillary venules (I). When vasculitis is idiopathic, it is called primary CSVV (4). There are several CSVV subtypes including immunoglobulin A vasculitis (Henoch-Schönlein purpura, HSP), urticarial vasculitis, and cryoglobulinemic vasculitis (4). Furthermore, the small vessel vasculitides are subdivided into antineutrophil cytoplasmic antibody (ANCA)-associated vasculitis which can involve not only small-sized vessels but also medium-sized vessels. The ANCA-associated vasculitides include microscopic polyangiitis, Churg-Strauss syndrome, and granulomatosis with polyangiitis (formerly called Wegener's granulomatosis) (5).

Cutaneous small vessel vasculitis almost always affects the small vessels of the skin, but sometimes extra cutaneous involvement may also occur. Moreover, CSVV findings may appear as cutaneous manifestations of large systemic vasculitides (6). The distinctive clinical feature is the palpable purpura usually involving the lower extremities (4). Although CSVV can be induced by a variety of causes including drugs, infections, connective tissue diseases, and malignancies, it can also be idiopathic $(3,6)$. The duration of the cutaneous lesions is uncertain. There are several treatment options, but symptomatic measures are enough for most of the cases.

Hence, the aim of the present study was to retrospectively analyze the medical records of patients with cutaneous LCV to determine demographic characteristics, etiological factors, clinical signs and symptoms, and treatment options in 57 patients.

\section{MATERIALS and METHODS}

In this cross-sectional study, the records of all patients from 2013 to 2017 with CSVV diagnosed clinically and/or histopathologically at the Department of Dermatology were reviewed. Data were collected from the hospital's database system retrospectively. Patients with diseases leading to bleeding diathesis, patients using anticoagulants, pregnant women, and patients who did not want to sign the informed consent form to allow the evaluation of information regarding their medical history were excluded from the study. The study was approved by the institutional ethics committee of Ankara Training and Research Hospital (Approval no:5/2019, Date:25.04.2019). All participants were informed about the study. Written informed consent was obtained from all of the patients.

Demographic characteristics including age and gender, disease history, possible etiological factors, accompanying conditions, and physical and dermatological examinations of all the patients were reviewed. Disease characteristics were evaluated carefully to determine the disease duration, cutaneous and systemic symptoms, etiologies including infections, drug usage, malignancy, connective tissue diseases, and other associated systemic disorders. Treatment modalities were also evaluated.

Laboratory tests and imaging studies were also collected. These include complete blood count, renal and liver function tests, urine analysis, stool for occult blood, C-reactive protein (CRP), erythrocyte sedimentation rate (ESR), antistreptolysin $O$ titer, antinuclear antibody, ANCA (both cytoplasmic ANCA: an- ti-proteinase-3 and perinuclear ANCA: anti-myeloperoxidase antibodies), serum complement fractions, cryoglobulin level, rheumatoid factor, human immunodeficiency virus, hepatitis B virus and hepatitis $C$ virus serology, chest $X$-ray, and abdominal ultrasonography. Two skin biopsies (punch biopsy, $4 \mathrm{~mm}$ ) were obtained from the lesions with <48-hour duration for routine histopathology and direct immunofluorescence (DIF). The histopathological confirmation of cutaneous LCV was made by the presence of a perivascular neutrophil-dominant inflammatory infiltrate, leukocytoclasia, fibrinoid necrosis, and extravasation of red blood cells into the vessel wall (I).

\section{Statistical Analysis}

All analyses were performed using the Statistical Package for the Social Sciences, version 16.00 (SPSS Inc.; Chicago, IL, USA). Normality of the data was tested. Continuous variables were expressed as mean \pm standard deviation, if they had a normal distribution or otherwise, and as median and minimum-maximum. Categorical variables were expressed as number (percentage). Chi-square test or Fisher's exact test was used to analyze categorical variables. Fisher's exact test was used when one or more cell counts were $<5$. A p value $<0.05$ was considered as statistically significant.

\section{RESULTS}

A total of 57 patients were included in the study. There were $39(68.4 \%)$ female and $18(31.6 \%)$ male patients. The femaleto-male ratio was 2.17. The median age of the patients was 55 (14-86) years. The median duration of the disease was I4 (2-120) days.

After all the patients were classified based on clinical findings and laboratory assessments, it was found that the majority of patients $(46,80.7 \%)$ had CSVV. This is a diagnosis of exclusion which can only be made after ruling out all other possibilities. The distribution of patients with small vessel vasculitides is presented in Table I. The etiological factors and associated conditions in our patients are shown in Table 2 . In most of the patients $(24,42 \%)$, the etiological cause could not be identified, and they were classified as primary CSVV.

TABLE I. The distribution of patients with small vessel vasculitides

$\begin{array}{lc}\text { Small vessel vasculitides } & \text { Patients (n) } \\ \text { Cutaneous small vessel vasculitis } & 46(80.7 \%) \\ \text { Primary CSVV } & 24(42 \%) \\ \text { Secondary CSVV } & 22(38.6 \%) \\ \text { Subtypes of CSVV } & \\ \text { Henoch-Schönlein purpura } & 4(7 \%) \\ \text { Urticarial vasculitis } & 4(7 \%) \\ \text { Cryoglobulinemic vasculitis } & 1(1.8 \%) \\ \text { ANCA-associated vasculitis } & \\ \text { Granulomatosis with polyangiitis } & 1(1.8 \%) \\ \text { c-ANCA vasculitis, not otherwise specified } & \text { I (I.8\%) }\end{array}$

ANCA: antineutrophil cytoplasmic antibody; c-ANCA: cytoplasmic antineutrophil cytoplasmic antibody; CSVV: cutaneous small vessel vasculitis 
The cutaneous symptoms and signs of LCV in our study group are presented in Table 3. Cutaneous lesions in 26 (45.6\%) patients were asymptomatic, but 18 (31.6\%) patients had pruritus, $10(17.6 \%)$ patients had pain associated with the lesions, and 10 (17.6\%) patients had burning sensation. Cutaneous lesions involved the lower extremities in $70.2 \%(40 / 57)$ of the patients. Moreover, $22(38.6 \%)$ patients had cutaneous lesions involving only the lower extremities. There were also 13 (22.2\%) patients with upper extremity lesions, 10 (17.5\%) patients with truncal lesions, and I (I.8\%) patient with mucosal lesions. Twenty (35.I\%) patients had generalized cutaneous lesions.

Extra cutaneous involvement (Table 4) was observed in 19 (33.3\%) patients. The most common extra cutaneous manifestation was joint involvement. In 15 cases, arthralgia was found in 12 patients, and arthritis was found in 3 patients (mostly involving the knee). Systemic involvement was detected in approximately one-third of the patients. Joint involvement manifests itself as arthritis and/or arthralgia. Gastrointestinal involvement results in abdominal pain and/or occult blood positivity. Renal involvement causes hematuria and/or proteinuria. Joint involvement was found in 15 (26.3\%) patients, gastrointestinal involvement was found in 9 (15.7\%) patients, and renal involvement was found in 12 (21\%) patients. Patients with renal involvement were consulted to the nephrology department for further evaluation. A 24-hour urine analysis revealed non-nephrotic range proteinuria. Therefore, renal biopsy was not performed, and regular follow-up was scheduled. The investigative profile of the patients is presented in Table 5. The most frequent three laboratory abnormalities were an elevated CRP (40 patients, 70.2\%), elevated ESR (33 patients, 57.9\%), and anemia (24 patients, 42.1\%). There is no relationship between high ESR, presence of leukocytosis, high CRP, and systemic involvement ( $p=0.569, p=0.095, p=0.370)$.

\begin{tabular}{|lc|}
\hline \multicolumn{2}{|l|}{ TABLE 2. Etiology and associated conditions of small vessel vasculitides } \\
\hline Etiology or association & Patients (n) \\
\hline Idiopathic & $25(43.9 \%)$ \\
Drug-induced & $10(17.5 \%)$ \\
Infection & $8(14 \%)$ \\
Drug and infection & $12(21 \%)$ \\
Connective tissue diseases & $7(12.3 \%)$ \\
(rheumatoid arthritis, dermatomyositis) & $4(3 / 1)(7 \%)$ \\
\hline Malignancy (solid/hematologic) & \\
\hline *Each patient may be listed in more than I category & \\
\hline
\end{tabular}

TABLE 3. Cutaneous manifestations of vasculitis

\begin{tabular}{|lc|}
\hline Cutaneous manifestations & Patients $(\mathbf{n})$ \\
\hline Palpable purpura & $21(36.8 \%)$ \\
Non-palpable purpura & $18(31.6 \%)$ \\
Ulcers & $12(21 \%)$ \\
Urticarial lesions & $2(3.5 \%)$ \\
Vesiculobullous lesions & $2(3.5 \%)$ \\
Nodules & $\mathrm{I}(1.8 \%)$ \\
Livedo reticularis & $\mathrm{I}(1.8 \%)$ \\
\hline
\end{tabular}

Two skin biopsies for routine histopathology and DIF examination were performed in $49(85.9 \%)$ patients. Histopathological examination of these patients revealed LCV in 45 (91.8\%) patients and nonspecific findings in $4(8.2 \%)$ patients. DIF examination was positive in $30(61.3 \%)$ patients. The most common deposit positivity was for $\mathrm{C} 3$ alone in 15 (30.61\%) patients, followed by $\lg \mathrm{M}$ and $\mathrm{C} 3$ in 9 (18.3\%) patients, IgA in 4 (8.16\%) patients, and $\lg G, \lg M$, and $C 3$ in $2(4.1 \%)$ patients.

Conventional therapeutic approach for the management of patients with CSVV was symptomatic, such as follow-up, elevation of extremities, application of topical corticosteroids, and/or systemic antihistamines. These first-line treatment options were used in 47 (82.5\%) patients. A specific treatment was initiated in I0 (17.5\%) patients, mainly for patients with a longer duration of CSVV and more severe clinical manifestations. Four (7\%) patients received systemic steroids, $3(5.3 \%)$ patients received colchicine, 2 (3.5\%) patients received dapsone, and I (I.8\%) patient received azathioprine therapy.

\section{DISCUSSION}

Leukocytoclastic vasculitis refers to the new name for a histopathological description that is neutrophilic inflammation of the postcapillary venules (I). A wide variety of clinical entities can lead to LCV in the skin. In this retrospective, cross-sectional study, we report findings in 57 patients with LCV.

\begin{tabular}{lc|}
$\begin{array}{l}\text { TABLE 4. Extra cutaneous associations of small vessel vasculitides in } \\
\text { the patients }\end{array}$ \\
\hline Extra cutaneous manifestations & Patients (n) \\
\hline None & $38(66.7 \%)$ \\
Arthralgia & $12(21 \%)$ \\
Arthritis & $3(5.3 \%)$ \\
Abdominal pain & $4(7 \%)$ \\
Fever & $4(7 \%)$ \\
Fatigue & $4(7 \%)$ \\
Nausea and vomiting & $2(3.5 \%)$ \\
Weight loss & $1(1.8)$ \\
\hline
\end{tabular}

TABLE 5. Laboratory evaluation of the patients

Test

Patients ( $n$ )

Elevated CRP $(>0.8 \mathrm{mg} / \mathrm{dL}) \quad 40(70.2 \%)$

Elevated ESR $(20 \mathrm{~mm} / \mathrm{h}) \quad 33(57.9 \%)$

Anemia $(\mathrm{Hb}<12 \mathrm{~g} / \mathrm{dL}) \quad 24(42.1 \%)$

Leukocytosis $\left(>10000 \mathrm{~mm}^{3}\right) \quad 16(28.1 \%)$

Elevated ASO $(>200 \mathrm{IU} / \mathrm{mL}) \quad 12(21 \%)$

$\begin{array}{ll}\text { Hematuria } & 12(21 \%)\end{array}$

Elevated liver function tests (ALT > $40 \mathrm{U} / \mathrm{L} ; \mathrm{AST}>33 \mathrm{U} / \mathrm{L}$ ) $\quad \|(19.3 \%)$

Rheumatoid factor $(>20 \mathrm{IU} / \mathrm{mL}) \quad 8(14 \%)$

$\begin{array}{ll}\text { ANA positivity } & 8(14 \%)\end{array}$

Hypocomplementemia (C3 79-152 mg/dL, C4 $16-38$ mg/dL) 6(I0.5\%)

Fecal occult blood $\quad 5(8.8 \%)$

ALT: alanine aminotransferase; ANA: antinuclear antibody; ASO: antistreptolysin O; AST: aspartate aminotransferase; CRP: C-reactive protein; ESR: erythrocyte sedimentation rate; $\mathrm{Hb}$ : hemoglobin 
In the present study, the median age of patients with LCV was 55 years, and the female-to-male ratio was 2.17. Chua et al. (7) also conducted a retrospective analysis of cutaneous vasculitis and reported that the female-to-male ratio is 2 in their study group. However, some other studies have found that the disorder was more common in men or equal in both sexes $(8,9)$.

Primary CSV V represented the majority of patients (42\%) with cutaneous LCV in our study, which is similar to the $40.7 \%$ incidence reported by Blanco et al. and $36.8 \%$ incidence reported by Al-Mutairi $(6,10)$. Tai et al. (II) evaluated 93 adult patients and reported that the most common small vessel vasculitis is cutaneous LCV (68 patients, 73.2\%) in their study group, followed by HSP (II patients, II.8\%), microscopic polyangiitis (5 patients, $5.4 \%$ ), septic vasculitis (4 patients, $4.3 \%$ ), Wegener's granulomatosis (2 patients, 2.2\%), Churg-Strauss syndrome (I patient, I.I\%), and essential cryoglobulinemic vasculitis (I patient, I.1\%), respectively. Blanco et al. (6) conducted a study involving 303 patients consisting of 172 adults and $13 \mathrm{I}$ children. The most common small vessel vasculitis in the study population was HSP (I55 patients, $51.6 \%$ ), followed by cutaneous LCV (84 patients, 27.7\%). The other diagnoses were polyarteritis nodosa ( 17 patients, 5.6\%), essential mixed cryoglobulinemia (II patients, 3.6\%), Wegener's granulomatosis (4 patients, $1.3 \%$ ), and Churg-Strauss syndrome (2 patients, $0.6 \%$ ), respectively. In our study of 57 patients, 46 (80.7\%) patients had CSVV (24 primary and 22 secondary), 4 (7\%) patients had HSP, 4 (7\%) patients had urticarial vasculitis, 2 (3.5\%) patients had ANCA vasculitis, and I (I.8\%) patient had cryoglobulinemic vasculitis. The difference in the incidence of the types of vasculitides in several studies might be explained by the use of different classification systems, such as the American College of Rheumatology criteria and the revised Chapel Hill Congress Conference nomenclature system that had variable definitions for vasculitides and vasculitic syndromes $(2,3)$. Moreover, the age range of the patients included in the studies might affect the incidence of vasculitides. HSP cases appeared to be more common in the studies that included a higher number of patients belonging to the pediatric population (6).

Although almost half of the cases with CSV $V$ are idiopathic, a detailed investigation for any underlying causes or associations is essential (I). CSV $\vee$ may be caused by infections (I5\%-20\%), autoimmune connective tissue diseases or inflammatory conditions (15\%-20\%), drugs (10\%-15\%), and hematologic or solid malignancies $(5 \%)(1,12)$. In our study group, the main cause was the presence of simultaneous drug usage and infection (I2 patients, $21 \%$ ). The second and third most common causes were drug usage alone (10 patients, $17.5 \%$ ) and having an infection alone ( 8 patients, 14\%) respectively. Drug- and infection-induced vasculitis was observed more frequently in our study group than in previous studies $(6,8,13)$. This may be due to the fact that patients applying to a tertiary institution (probably after primary practices) were more likely to be complicated by an infection and consequently had increased use of medication.

Palpable purpura (2l patients, 36.8\%) involving the lower extremities (40 patients, 70.2\%) was the most commonly observed cutaneous manifestation of LCV in the present study, in accordance with the previous studies $(14,15)$. It was assumed as the most sensitive diagnostic sign for cutaneous LCV (16). Lesions may cause pruritus, pain, and burning as mentioned in our study (4).
Leukocytoclastic vasculitis primarily involves the small caliber blood vessels of the skin, but in approximately $50 \%$ of the patients, the small vessels of the joints, gastrointestinal tract, kidneys, muscles, lungs, and peripheral nerves could be involved, leading to multisystem organ involvement (17). We observed systemic involvement in approximately one-third of our patients. Arthralgia and arthritis, namely joint involvement, were the most frequent extra cutaneous findings of our study. The other frequent extra cutaneous findings were renal and gastrointestinal involvements, respectively. In line with the findings of our study, several studies evaluating the sites of involvement in LCV reported that the most frequent non-cutaneous finding was joint involvement (14, 18-20).

In the present study, the most frequent laboratory abnormality was an elevated CRP level, followed by elevated ESR and anemia. However, there is no relationship between high ESR, presence of leukocytosis, high CRP, and presence of systemic involvement. Most of the studies revealed that ESR was the most frequent pathological laboratory finding (II, 18, 20), whereas some of them also indicated a relationship between elevated ESR and systemic involvement (I4).

The natural course of LCV is usually benign and self-limiting, so symptomatic measures are the first-line treatment option. Follow-up, topical corticosteroids, and systemic antihistamines are sufficient to control the symptoms in most of the cases. If the disease is persistent and/or recurrent, drugs, such as dapsone, colchicine, and pentoxifylline, are used. If there is a moderate-to-severe disease accompanied by vesicles, ulcers, and nodules, systemic steroids and immunosuppressive drugs, such as azathioprine, methotrexate, cyclosporine, and cyclophosphamide, are given (10). In our study group, first-line treatment options were enough to control the symptoms of $47(82.5 \%)$ patients. A total of 5 patients with a longer disease duration and more severe clinical manifestations had received immunosuppressive treatment. Four (7\%) patients received systemic steroids, I (1.8\%) patient received azathioprine therapy, 3 (5.3\%) patients received colchicine, and 2 (3.5\%) patients received dapsone. Cakiter et al. (18) evaluated treatment options in 75 patients and reported that symptomatic treatment is enough in the management of $46(61.3 \%)$ patients, whereas the rest of the patients needed specific treatment options, such as systemic steroids (12 patients, 16\%), colchicine (9 patients, 12\%), dapsone (7 patients, 9.3\%), and azathioprine (I patient, I.3\%). Boviller et al. (2I) studied II 2 patients with LCV and reported that a specific treatment is initiated in $4 \mathrm{I}(36.6 \%)$ patients and those were the secondary cases with an underlying cause. In their study, the most commonly preferred treatment options were prednisone (70.7\%), hydroxychloroquine (14.6\%), and colchicine (12.2\%). LCV treatment is initiated based on the severity of disease and end organ involvement. Therefore, the frequency of the use of treatment options varies between studies, but aggressive treatment is not necessary most of the time because of the benign course of the disease.

In conclusion, LCV is a clinicopathological term gathering several vasculitis entities together. LCV affected patients with a median age of 55 years, and there was a female preponderance in the present study. LCV classically presented as symmetric palpable purpura of the lower extremities. The disease was 
frequently idiopathic, but the most commonly identified underlying cause was the presence of simultaneous drug usage and infection. An elevated CRP was the most common laboratory finding. Symptomatic treatment options are sufficient for most of the cases due to the benign, self-limiting course of the disease. In clinical practice, it is important to keep in mind that although LCV is usually confined to the blood vessels of the skin, a detailed evaluation of the patients is required to detect the underlying causes and systemic involvement.

Ethics Committee Approval: Ethics committee approval was received for this study from the ethics committee of Ankara Training and Research Hospital (Approval no:5/2019, Date:25.04.2019).

Informed Consent: Written informed consent was obtained from the patients who participated in this study.

Peer-review: Externally peer-reviewed.

Author Contributions: Concept - E.K., N.K.; Design - P.Ö.C.., E.K.; Supervision - N.K.; Resources - E.K.; Materials - E.K.; Data Collection and/or Processing - E.K., P.Ö.C.; Analysis and/or Interpretation - P.Ö.C.., N.K.; Literature Search - P.Ö.C.; Writing Manuscript - P.Ö.C., N.K.; Critical Review - P.Ö.C., N.K., E.K.

Conflict of Interest: The authors have no conflicts of interest to declare.

Financial Disclosure: The authors declared that this study has received no financial support.

\section{REFERENCES}

I. Chung L, Kea B, Fiorentino Df. Cutaneous Vasculitis. In: Bolognia Jl, Jorizzo Jl, Rapini Rp, Editors. Dermatology. 2nd Ed. St. Louis (Mo): Elsevier Limited; 2008. P. 347-67.

2. Hunder GG, Arend WP, Bloch DA, Calabrese LH, Fauci AS, Fries JF, et al. The American College of Rheumatology 1990 criteria for the classification of vasculitis introduction. Arthritis Rheum 1990; 33(8): 1065-7. [CrossRef]

3. Jennette JC, Falk RJ, Bacon PA, Basu N, Cid MC, Ferrario F, et al. 2012 Revised international chapel hill consensus conference nomenclature of vasculitides. Arthritis Rheum 2013; 65(I): I-II. [CrossRef]

4. Goeser MR, Laniosz $\bigvee$, Wetter DA. A Practical Approach to the Diagnosis, Evaluation, and Management of Cutaneous Small-Vessel Vasculitis. Am J Clin Dermatol 2014; 15(4): 299-306. [CrossRef]

5. Shavit E, Alavi A, Sibbald RG. Vasculitis-what do we have to know? a review of literature. Int J Low Extrem Wounds 2018; 17(4): 218-26. [CrossRef]
6. Blanco R, Martinez-Taboada VM, Rodriguez-Valverde V, Garcia-Fuentes M. Cutaneous vasculitis in children and adults. Associated diseases and etiologic factors in 303 patients. Medicine (Baltimore) 1998; 77(6): 403-18. [CrossRef]

7. Chua SH, Lim JT, Ang CB. Cutaneous vasculitis seen at a skin referral centre in Singapore. Singapore Med J 1999; 40(3): 147-50.

8. Garcia-Porrua C, Gonzalez-Gay MA, Lopez-Lazaro L. Drug associated cutaneous vasculitis in adults in northwestern Spain. J Rheumatol 1999; 26(9): 1942-4.

9. Arora A, Wetter DA, Gonzalez-Santiago TM, Davis MD, Lohse CM. Incidence of leukocytoclastic vasculitis, 1996 to 20I0: a population-based study in olmsted county, Minnesota. Mayo Clin Proc 2014; 89(II): 1515-24. [CrossRef]

10. Al-Mutairi N. Spectrum of cutaneous vasculitis in adult patients from the Farwaniya region of Kuwait. Med Princ Pract 2008; 17(I): 43-8. [CrossRef]

II. Tai YJ, Chong AH, Williams RA, Cumming S, Kelly RI. Retrospective analysis of adult patients with cutaneous leukocytoclastic vasculitis. Australas J Dermatol 2006; 47(2): 92-6. [CrossRef]

12. Russell JP, Gibson LE. Primary cutaneous small vessel vasculitis: approach to diagnosis and treatment. Int J Dermatol 2006; 45(I): 3-13. [CrossRef]

13. Jessop SJ. Cutaneous leucocytoclastic vasculitis: a clinical and aetiological study. Br J Rheumatol 1995; 34(I0): 942-5. [CrossRef]

14. Sais G, Vidaller A, Jucgla A, Servitje O, Condom E, Peyri J. Prognostic factors in leukocytoclastic vasculitis: a clinicopathologic study of 160 patients. Arch Dermatol 1998; 134(3): 309-15. [CrossRef]

15. Hodge SJ, Callen JP, Ekenstam E. Cutaneous leukocytoclastic vasculitis: correlation of histopathological changes with clinical severity and course. J Cutan Pathol 1987; 14(5): 279-84. [CrossRef]

16. Calabrese LH, Michel BA, Bloch DA, Arend WP, Edworthy SM, Fauci $A S$, et al. The American College of Rheumatology 1990 criteria for the classification of hypersensitivity vasculitis. Arthritis Rheum 1990; 33(8): II08-13. [CrossRef]

17. Jennette JC, Falk RJ. Small-vessel vasculitis. N Engl J Med 1997; 337(21): 1512-23. [CrossRef]

18. Cakiter AU, Kucuk OS, Ozkaya DB, Topukcu B, Onsun N. Demographic characteristics, aetiology, and assessment of treatment options in leukocytoclastic vasculitis. Postepy Dermatol Alergol 2017; 34(2): 104-9. [CrossRef]

19. Ekenstam E, Callen JP. Cutaneous leukocytoclastic vasculitis. clinical and laboratory features of 82 patients seen in private practice. Arch Dermatol 1984; 120(4): 484-9. [CrossRef]

20. Sahin Eb, Hapa a, Elçin G, Karaduman a, Evans Ersoy S, Atakan N, et Al. Leukocytoclastic vasculitis retrospective analysis of 60 patients. Turk Dermatoloji Dergisi 20II; 5: 85-9I. [CrossRef]

21. Bouiller K, Audia S, Devilliers H, Collet E, Aubriot MH, Leguy-Seguin $\checkmark$, et al. Etiologies and prognostic factors of leukocytoclastic vasculitis with skin involvement: a retrospective study in II2 patients. Medicine (Baltimore) 2016; 95(28): e4238. [CrossRef] 